\title{
Eating habits and factors affecting food choice of adolescents living in rural areas
}

\author{
Alexandra Bargiota, ${ }^{1}$ Maria Delizona, ${ }^{1}$ Andreas Tsitouras, ${ }^{2, \dagger}$ Georgios N. Koukoulis ${ }^{1}$ \\ ${ }^{1}$ Department of Endocrinology and Metabolic Diseases, University Hospital of Larissa, Medical School, University of \\ Thessaly, ${ }^{2}$ Department of Dietetics and Nutritional Science, Harokopio University, Athens, Greece \\ †Deceased
}

\begin{abstract}
OBJECTIVE: To establish factors that affect food choices among adolescents living in rural areas and to identify their food choices. DESIGN: A random sample of adolescents living in a Greek rural area $(n=382)$ aged 12-18 years were individually interviewed. Food consumption was assessed by a semi-quantitative food-frequency questionnaire and adherence to the Mediterranean diet was evaluated using the KIDMED questionnaire. Information was collected regarding self-perceived body size, dieting, dietary knowledge, parental control, meal and snack frequency, eating out of home, eating takeaways and precooked meals, eating from the school canteen. RESULTS: Body image concerns, dieting, education about food, parental control, maternal education level and eating with family and peers are factors that were found to affect food choices in this group of Greek adolescents. The adherence to the Mediterranean diet was low (KIDMED index was 4.5 \pm 2.7 ). Regular family meals at home were frequent in this group and $99 \%$ of the adolescents ate lunch daily at home. Eating out with peers and eating from the school canteen was related with higher consumption of 'junk type of food'. Girls and younger adolescents and those whose mothers had a higher education level seem to make healthier choices. CONCLUSIONS: Factors such as personal issues, family and peer pressure significantly affect food choices among adolescents living in a Greek rural area and highlight the importance of implementing multilevel strategies to promote healthy eating among adolescents.
\end{abstract}

Key words: Adolescents, Eating habits, Family, Food labels, Peers, School canteen

\section{INTRODUCTION}

Rates of overweight and obesity have increased dramatically among adolescents and lifestyle changes

Address for correspondence:

Dr. A. Bargiota, Lecturer in Endocrinology, University

Hospital of Larissa, Biopolis Larissa, 41100 Greece,

E-mail: abargio@yahoo.gr

Received 02-07-2012, Accepted 07-03-2013 seem to be the main contributor to this change..$^{1-3}$ Adolescent obesity is not only capable of affecting adolescents' current health but also has an impact on later life independently of weight status in adulthood. Dietary intake and nutrition behaviours at this period of life influence lifelong eating habits and contribute to the development of risk factors for disease in adulthood. ${ }^{4}$ Therefore, identification of the reasons behind food choices could help to reduce the burden of several diseases. 
Food choice in general is a complex process that depends on culture and can be influenced by different factors such as personal, social, economic and emotional. Teenagers make many more choices for themselves than they did as children. Moreover, since eating is a social act, social networks and family can affect their food choices even more..$^{5}$ In addition, globalization is eroding traditional foods and offering more food choice and availability, all of which could influence their eating patterns. ${ }^{6,7}$

Recent studies in Greece have shown that food habits in adolescents have changed and low adherence rates to the traditional Mediterranean diet have been observed, particularly in Greek children and adolescents. ${ }^{8-10}$ The majority of these studies focus on the evaluation of the nutrition status of adolescents; however, there are very limited data on factors that influence food choice in this age group in Greece. There is also little information about the meal and snack frequency of Greek adolescents as well as the places where adolescents usually eat and the timing of their meals. The purpose of the present study was to examine such eating behaviours in a cohort of Greek adolescents and the factors influencing their food choices and habits.

\section{SUBJECTS AND METHODOLOGY}

\section{Subjects}

Data were collected from 382 high school students from May to June of 2008. Stratified and random sampling from three different schools of a rural Greek area (Nea Moudania, Halkidiki) was applied. Validated responses were obtained from a total of 350 adolescents (161 boys, $46 \%$ and 189 girls, $54 \%$ ), aged 12-18 years. Adolescents were further divided into two age groups: younger adolescents (12-14 years) attending the Greek "gymnasium" (first three classes of high school) and older adolescents (15-18 years), attending the Greek "lyceum" (last three classes of high school).

\section{Assessment of dietary intake and eating behaviour}

Usual dietary intake during the month preceding enrolment was assessed with the use of a semiquantitative food-frequency questionnaire including foods and beverages commonly consumed in Greece adapted to the adolescents' preferences and has been previously validated. ${ }^{11,12}$ The KIDMED questionnaire was used to assess whether the adolescents were keeping to the Mediterranean diet. The KIDMED questionnaire was recently developed to assess the compliance of young people (2-24 years) to the Mediterranean diet, and higher values of this index suggest greater nutritional adequacy, especially in vitamins and minerals. ${ }^{6,8}$ Additionally, the following factors were examined and analyzed: self-perceived body size, dieting, dietary knowledge, parental control, meal and snack frequency, eating out of home, eating takeaways and precooked meals, eating from the school canteen. Data were collected by a trained dietician with the help of the parents during a personal interview. Parents also provided information regarding their educational level which was divided into 3 groups: low [illiteracy, primary, junior middle school (9 y)]; medium [senior middle school (10-12 y)]; and high [university or higher education (13 y)]. ${ }^{8}$ The study protocol was approved by the local educational board and parent consent was obtained in advance for each participant.

\section{Statistical analysis}

Continuous variables are presented as means \pm $\mathrm{SD}$ and categorical variables as absolute frequencies. Descriptive statistics were used to describe dietary practices. Multivariate linear and logistic regression analyses were used to assess the determinant role of sex, age, dietary knowledge and parents' education level and parental control on dietary practices. The results from regression models are presented as standardized $\beta$ coefficients. The chi square test was used to test for differences in categorical variables. The level of significance was defined at $P<0.05$. Data were analyzed using the Statistical Package for the Social Sciences (SPSS13) software.

\section{RESULTS}

\section{The overall response rate to the survey was $\mathbf{9 1 . 6 \%}$ self-perceived body size}

Of the boys participating, $22.4 \%$ thought they were underweight, $13 \%$ too heavy and $64.6 \%$ 'about right'. Among the girls the corresponding proportions were not very different: $20.1 \%$ thought they were too light, $15.4 \%$ too heavy and $64.5 \%$ 'about right'. $29 \%$ of the 
boys and $30 \%$ of the girls replied that they were on a slimming diet in order to lose weight.

\section{Dietary knowledge}

Of the participants, $72 \%$ reported receiving sufficient education about reading and understanding food labels with information on ingredients. Girls and older adolescents read significantly more information on food labels than boys and younger teenagers $(\mathrm{p}<0.001, \beta=0.071 ; \mathrm{p}<0.001, \beta=0.202$, respectively). When adolescents buy food by themselves they are interested in low total calories, low quantity of fat and the expiry date of the product. When they shop with their parents they pay attention to the type and the percentage of the additives and conservatives of the product, the country which has produced it and its expiry date (Figure 1). $75 \%$ replied that they knew what the Mediterranean diet and its main components are. Approximately $22 \%$ of the adolescents responded that their food selection was based solely on health concerns, $17 \%$ only on taste regardless of

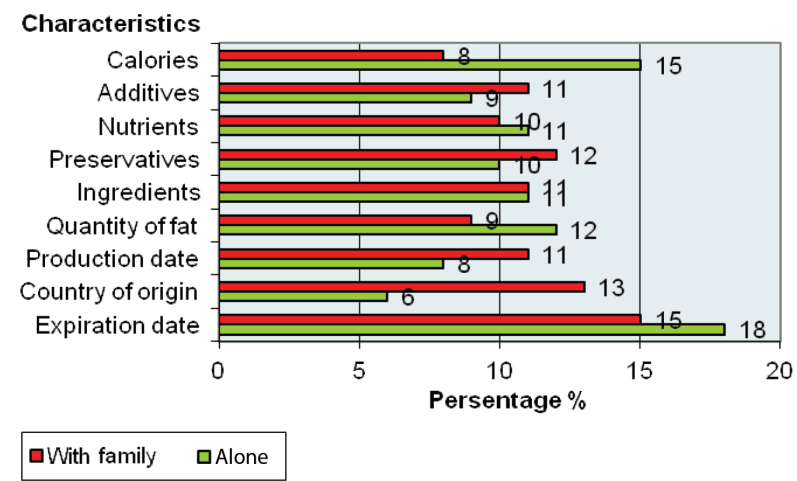

Figure 1. Food label information that the adolescents are interested in when they shop alone and with their parents. its impact on their health and $53 \%$ on both health concern and taste.

\section{Parental control}

Of the participants, $93 \%$ replied that their parents control their food choices: $20 \%$ very much, $42 \%$ quite a lot, $21 \%$ moderately and $10 \%$ not very much. Parental control was greater among the younger adolescents $(\mathrm{p}<0.01 \beta=-0.144)$.

\section{Meal and snack frequency}

Table 1 displays the number of meals and snacks that adolescents ate on a daily basis. Compared to boys, significantly fewer girls ate dinner ( $84 \%$ boys, $71 \%$ girls, $\mathrm{p}<0.04)$ and a snack before bed $(\mathrm{p}<0.035)$. Dinner time was fixed, between 20.00 and $21.00 \mathrm{pm}$ for $35 \%$ of the adolescents.

\section{Eating out}

Figure 2 shows the places where adolescents go to eat when they go out with their parents and with

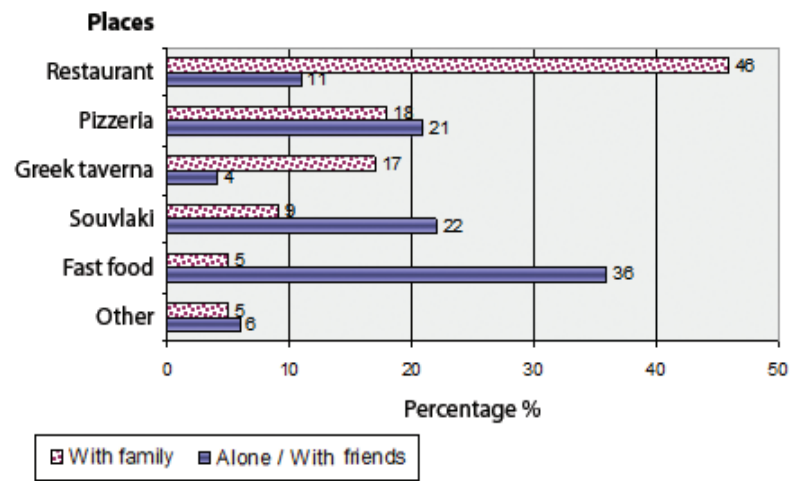

Figure 2. Places where adolescents ate when they went out with parents and friends.

Table 1. Frequency of daily meals and snacks of Greek adolescents. Results are given as absolute numbers (n) and percentages (\%)

\begin{tabular}{|c|c|c|c|c|c|c|c|c|c|c|c|c|}
\hline \multirow[b]{3}{*}{ Meals } & \multicolumn{4}{|c|}{ Boys } & \multicolumn{4}{|c|}{ Girls } & \multicolumn{4}{|c|}{ Total } \\
\hline & \multicolumn{2}{|c|}{ Eat } & \multicolumn{2}{|c|}{ Don't eat } & \multicolumn{2}{|c|}{ Eat } & \multicolumn{2}{|c|}{ Don't eat } & \multicolumn{2}{|c|}{ Eat } & \multicolumn{2}{|c|}{ Don't eat } \\
\hline & $\mathbf{n}$ & $\%$ & $\mathbf{n}$ & $\%$ & $\mathbf{n}$ & $\%$ & n & $\%$ & $\mathbf{n}$ & $\%$ & n & $\%$ \\
\hline Breakfast & 124 & 77 & 37 & 23 & 134 & 71 & 55 & 29 & 258 & 74 & 92 & 26 \\
\hline Midmorning snack & 70 & 44 & 90 & 56 & 82 & 43 & 107 & 57 & 152 & 43 & 198 & 57 \\
\hline Lunch & 159 & 99 & 2 & 1 & 188 & 99 & 1 & 1 & 347 & 99 & 3 & 1 \\
\hline Afternoon snack & 92 & 57 & 69 & 43 & 101 & 53 & 88 & 47 & 193 & 55 & 157 & 45 \\
\hline Dinner & 136 & 84 & 25 & 16 & 135 & 71 & 54 & 29 & 271 & 77 & 79 & 23 \\
\hline Before bed snack & 24 & 15 & 137 & 85 & 12 & 6 & 177 & 94 & 36 & 10 & 314 & 90 \\
\hline
\end{tabular}


their peers. $53 \%$ of the adolescents answered that they ate out once a week and $21 \%$ ate out twice a week. When they ate out with the family they went to restaurants $(46 \%)$, a pizzeria $(18 \%)$ or to a traditional Greek tavern $(17 \%)$ and consumed significantly more pasta $(\mathrm{p}<0.005 \beta=0.015)$ and dairy products $(\mathrm{p}<0.01, \beta=0.084)$ but less vegetables $(\mathrm{p}<0.005$ $\beta=-0.092)$. However, when they ate out with peers or alone they went to fast food places $(36 \%)$ or ate traditional Greek fast-food [souvlaki, gyros (22\%)] and pizza $(21 \%)$. Boys ate out significantly more frequently than girls $(\mathrm{p}<0.005 \beta=0.219)$. Eating out was inversely related to the mother's age: the younger the mother, the higher the frequency of eating out $(\mathrm{p}<0.005 \beta=-0.19)$.

\section{Takeaways and precooked meals}

Of our adolescents, $77 \%$ replied that they ate takeaways at home, $45 \%$ of them once a week and $21 \%$ of them twice a week. For takeaways, pizza was their first preference, followed by kebabs and burgers. Very rarely the takeaway was a cooked meal and salad. Eating takeaways was positively related to the mother's educational level $(\mathrm{p}=0.044, r=0.108)$.

Consumption of precooked meals at home in the present group was low, with only $12 \%$ of the participants eating a precooked meal at home once a week.

\section{School canteen}

In this survey $83 \%$ of the adolescents replied that they bought food from the school canteen: $42 \%$ did so daily, $13 \%$ three times a week and $12 \%$ twice a week. Foods the most frequently consumed from the canteen were toast with cheese and ham (36\%), soft drinks and beverages (32\%) and sweets (20\%). $40 \%$ of the adolescents replied that they were very satisfied with the quality of the food of the school canteen and $30 \%$ moderately so. Eating from the school canteen was negatively related to the number of details that adolescents read on food labels $(\mathrm{p}=0.027 \beta=-0.092)$.

In addition, $47 \%$ of the participants replied that they ate at school a snack prepared at home, 24\% of them on a daily basis and $50 \%$ a few times a week. Girls and younger adolescents ate a home prepared snack at school more often than boys and the older adolescents $(\mathrm{p}<0.05 \beta=0.053$ and $\mathrm{p}<0.001$ $\beta=0.265$, respectively). Bringing a home prepared snack to school was inversely related to parental control $(\mathrm{p}<0.001 \beta=-0.207)$ and was a significant positive determinant for the number of elements that adolescents read on food labels $(\mathrm{p}<0.001 \beta=0.123)$.

Table 2 shows the KIDMED test and index in our sample. The KIDMED index score was $4.5 \pm 2.7$. Optimal KIDMED score $(>8)$ was found in $16 \%$ of the adolescents, average (4-7) in $45 \%$ of the adolescents and poor $(<3)$ in $39 \%$ of them. The score was higher in females than in males $(\mathrm{p}=0.007 \beta=0.078)$ and younger adolescents than the older ones $(p=0.028$ $\beta=-0.186$ ). Additionally, higher maternal education level was associated with significantly higher KIDMED score $(\mathrm{p}=0.037 \beta=0.027)$.

Table 3 indicates the weekly consumption of the different food groups. Girls consumed significantly more fruits $(\mathrm{p}=0.033 \beta=0.234)$ and vegetables $(\mathrm{p}=0.004 \beta=0.232)$ compared to boys. Twenty-nine percent $(29 \%)$ of the teenagers consumed alcohol once a week and $16 \%$ twice to three times a week.

\section{DISCUSSION}

In this study, we have examined the factors that affect food selections of a cohort of Greek adolescents and their eating behaviours and habits. Our data showed that many of the adolescents felt they were too heavy, this suggesting an awareness of their shape or image. About one third of them reported trying to lose weight by following a low fat diet, further indicating a concern about their body image. Previous studies indicated that approximately one out of five to six female adolescents face a risk for disordered attitudes regarding body image and food. ${ }^{10,13}$ Our data indicated an increased level of adolescents on a slimming diet, identical for boys and girls, which might be associated with the increasing levels of childhood obesity in Greece. In addition, in our study boys and girls up to the age of 18 years were included, among whom an increasing prevalence of dieting with age has previously been observed. ${ }^{10}$ Other possible explanations that can be offered are increasing family and media influences, peer pressure and personal issues during the critical time of puberty ${ }^{5,14-16}$ in a transition from traditional Greek to a more Westernized environment. ${ }^{8}$ It will be interesting to observe from future data collections whether this concern with body image will alter with age. 
Table 2. KIDMED test and KIDMED index in Greek adolescents $(n=350)$ aged 12-18 years, by gender

\begin{tabular}{|c|c|c|c|c|}
\hline \multicolumn{2}{|l|}{ KIDMED index } & Male $(\%)$ & Female $(\%)$ & Total $(\%)$ \\
\hline \multicolumn{2}{|l|}{ Fruit or fruit juice daily } & 45 & 58 & 51.7 \\
\hline \multicolumn{2}{|l|}{ Second serving of fruit daily } & 36 & 48 & 42 \\
\hline \multicolumn{2}{|l|}{ Fresh or cooked vegetables daily } & 22 & 40 & 32 \\
\hline \multicolumn{2}{|c|}{ Fresh or cooked vegetables $>1$ /day } & 16 & 34 & 25 \\
\hline \multicolumn{2}{|c|}{ Regular fish consumption (at least 2-3/week) } & 55 & 60 & 57.4 \\
\hline \multicolumn{2}{|c|}{$>1 /$ week fast-food (hamburger)restaurant } & 34 & 50 & 42.9 \\
\hline \multicolumn{2}{|c|}{ Pulses $>1 /$ week } & 55 & 63 & 59.7 \\
\hline \multicolumn{2}{|c|}{ Pasta or rice almost daily ( $\geq 5 /$ week) } & 34 & 39 & 36.3 \\
\hline \multicolumn{2}{|c|}{ Cereal or cereal product for breakfast } & 63 & 45 & 53.4 \\
\hline \multicolumn{2}{|c|}{ Regular nut consumption (at least 2-3/week) } & 13 & 17 & 15.4 \\
\hline \multicolumn{2}{|c|}{ Use of olive oil at home } & 96 & 97 & 96.6 \\
\hline \multicolumn{2}{|l|}{ No breakfast } & 23 & 29 & 26.3 \\
\hline \multicolumn{2}{|l|}{ Dairy product for breakfast } & 42 & 46 & 44 \\
\hline \multicolumn{2}{|c|}{ Commercially baked goods or pasties for breakfast } & 76 & 79 & 77.7 \\
\hline \multicolumn{2}{|c|}{ Two yoghurts and/or 40 gr cheese daily } & 50 & 47 & 48 \\
\hline \multicolumn{2}{|c|}{ Sweets and candy several times a day } & 85 & 80 & 82.6 \\
\hline \multicolumn{2}{|c|}{ KIDMED INDEX } & Poor $(\leq 3)$ & Average (4-7) & Good $(\geq 8)$ \\
\hline \multirow[t]{2}{*}{ Sex } & Male & 52 & 41 & 7 \\
\hline & Female & 39 & 50 & 11 \\
\hline \multirow[t]{3}{*}{ Mother's education Level (\%) } & Low & 7 & 3 & 10 \\
\hline & Medium & 61 & 57 & 58 \\
\hline & High & 32 & 40 & 32 \\
\hline \multirow[t]{3}{*}{ Father's education Level (\%) } & Low & 11 & 6 & 10 \\
\hline & Medium & 51 & 62 & 45 \\
\hline & High & 38 & 32 & 45 \\
\hline
\end{tabular}

Table 3. Weekly consumption of the different food groups and alcohol by the Greek adolescents $(n=350)$ aged $12-18$ years. Results are given by gender as percentages $(\%)$ and times per week $(\mathrm{t} / \mathrm{w})$

\begin{tabular}{|c|c|c|c|c|c|c|c|c|c|c|c|c|c|c|c|}
\hline \multirow{2}{*}{$\begin{array}{l}\text { Food item } \\
\text { or } \\
\text { Food group }\end{array}$} & \multicolumn{5}{|c|}{ Boys } & \multicolumn{5}{|c|}{ Girls } & \multicolumn{5}{|c|}{ Total } \\
\hline & $\begin{array}{c}\text { Never } \\
(\%)\end{array}$ & $\begin{array}{c}1 \mathrm{t} / \mathrm{w} \\
(\%)\end{array}$ & $\begin{array}{c}2-3 \mathrm{t} / \mathrm{w} \\
(\%)\end{array}$ & $\begin{array}{c}4-5 \mathrm{t} / \mathrm{w} \\
(\%)\end{array}$ & $\begin{array}{c}6-7 \mathrm{t} / \mathrm{w} \\
(\%)\end{array}$ & $\begin{array}{c}\text { Never } \\
(\%)\end{array}$ & $\begin{array}{c}1 \mathrm{t} / \mathrm{w} \\
(\%)\end{array}$ & $\begin{array}{c}2-3 \mathrm{t} / \mathrm{w} \\
(\%)\end{array}$ & $\begin{array}{c}4-5 \mathrm{t} / \mathrm{w} \\
(\%)\end{array}$ & $\begin{array}{c}6-7 \mathrm{t} / \mathrm{w} \\
(\%)\end{array}$ & $\begin{array}{c}\text { Never } \\
(\%)\end{array}$ & $\begin{array}{c}1 \mathrm{t} / \mathrm{w} \\
(\%)\end{array}$ & $\begin{array}{c}2-3 \mathrm{t} / \mathrm{w} \\
(\%)\end{array}$ & $\begin{array}{c}4-5 \mathrm{t} / \mathrm{w} \\
(\%)\end{array}$ & $\begin{array}{c}6-7 \mathrm{t} / \mathrm{w} \\
(\%)\end{array}$ \\
\hline Olive oil & 4 & 4 & 14 & 19 & 59 & 3 & 6 & 17 & 26 & 48 & 3 & 5 & 16 & 23 & 53 \\
\hline Fruits & 1 & 10 & 13 & 26 & 44 & 0 & 7 & 15 & 20 & 58 & 0 & 9 & 17 & 23 & 51 \\
\hline Vegetables & 3 & 12 & 28 & 35 & 22 & 3 & 3 & 15 & 32 & 41 & 3 & 10 & 21 & 34 & 32 \\
\hline Pasta / rise & 1 & 21 & 44 & 30 & 4 & 1 & 12 & 43 & 31 & 7 & 1 & 16 & 46 & 31 & 6 \\
\hline Cheese & 7 & 4 & 14 & 26 & 49 & 2 & 6 & 22 & 24 & 46 & 4 & 5 & 18 & 25 & 48 \\
\hline Pulse & 11 & 34 & 37 & 9 & 9 & 5 & 31 & 41 & 19 & 4 & 8 & 33 & 39 & 14 & 6 \\
\hline Bread & 4 & 8 & 11 & 14 & 63 & 7 & 11 & 19 & 17 & 46 & 6 & 10 & 15 & 16 & 53 \\
\hline Red meat & 7 & 31 & 32 & 17 & 13 & 4 & 32 & 40 & 19 & 5 & 5 & 31 & 37 & 18 & 9 \\
\hline Chicken & 10 & 35 & 30 & 14 & 11 & 12 & 33 & 33 & 17 & 5 & 11 & 35 & 32 & 15 & 7 \\
\hline Potatoes & 2 & 11 & 35 & 31 & 21 & 2 & 20 & 36 & 23 & 19 & 2 & 16 & 35 & 27 & 20 \\
\hline Dairy products & 4 & 5 & 20 & 29 & 42 & 0 & 9 & 17 & 28 & 46 & 2 & 7 & 19 & 29 & 43 \\
\hline Fishes & 10 & 35 & 37 & 11 & 7 & 5 & 35 & 32 & 20 & 8 & 7 & 35 & 34 & 16 & 8 \\
\hline Sweets & 3 & 22 & 34 & 20 & 15 & 6 & 28 & 32 & 14 & 20 & 7 & 25 & 34 & 17 & 17 \\
\hline Eggs & 3 & 40 & 36 & 10 & 5 & 12 & 41 & 32 & 11 & 4 & 11 & 41 & 34 & 10 & 4 \\
\hline Alcoholic drinks & 34 & 34 & 17 & 4 & 11 & 48 & 25 & 15 & 8 & 4 & 41 & 29 & 16 & 7 & 7 \\
\hline
\end{tabular}

t/w: time/week 
Furthermore, our data indicate that adolescents' concerns about their body image are an important contributing factor in choosing food. Interestingly in this study, although the majority of the adolescents replied that both taste and health were important in choosing food, when it came to food selection, their food choices were based on its content. When adolescents bought food by themselves, they focused mainly on information about low calories and low fat percentage on food labels. Perhaps adolescents desire to be healthy but seem not necessarily to be concerned with their health outcomes at this point in their lives. Previous studies that evaluated food habits in adolescents also discovered low health concerns in adolescents' food choices. ${ }^{17,18}$

Knowledge about food is considered to be beneficial not only for food choice and healthy weight reduction but also as concerns its long-term implications for general health. Food label reading has previously been used as a tool for nutritional education..$^{19}$ In the present study, girls and older adolescents seem to be better educated as they are interested in having more information on food labels when they buy food and are thus more aware of its implication for weight and health. In addition, although the majority of our sample replied that they had been educated and knew what the Mediterranean diet is, they did not seem to follow it. Our data indicated a low to moderate adherence to the Mediterranean diet, a finding that is in line with previous data from Greece and other Mediterranean countries. ${ }^{6-8,20,21}$ It follows that teenagers, despite being generally well informed about healthy eating, do not often translate this knowledge into their daily lives. Other studies have also shown that nutrition label reading and food education do not translate into healthier diet in adolescents. ${ }^{22,23}$ Therefore, it might be necessary for intervention programmes designed for adolescents to focus not only on education on healthy food eating but also on behaviour changes. These programmes should also take into account the adolescents' lifestyle and social environment. Eating is a social event and thus the presence of family and friends inevitably can affect food choices. ${ }^{24}$

Our adolescents ate with their families frequently, which is in agreement with other data. ${ }^{5,25}$ Lunch has traditionally been the main family meal in Greece and almost all of the participants (99\%) ate lunch daily with their families. Approximately two thirds of them had daily breakfast and dinner at home. These regular family meals may contribute to the formation of healthful eating habits and could serve as role models for healthy food choices. ${ }^{26-28}$ Moreover, in the current study, the majority of adolescents acknowledged that their parents controlled their food choices. Parental control was greater in younger adolescents than the older ones who probably at this age are starting to be more independent and to make their own choices. Younger adolescents had a significantly higher KIDMED score than the older ones and possibly the higher level of parental control in younger adolescents has contributed to that, although overall no relation was found between parental control and KIDMED score. Parental education has also been shown in large epidemiological studies to have a positive independent effect on the quality of children's diet. ${ }^{8,29-31}$ Our data have also shown that the higher the maternal education level, the better the KIDMED score. It has been speculated that higher parental education level may be related to higher income and thus greater availability of healthy foods, increased nutrition knowledge or increased motivation to follow a healthy lifestyle. In contrast with previous studies conducted in Greece, ${ }^{8}$ our data have shown no significant association between the father's educational level and the KIDMED score. The data in the present study were collected from a mainly rural area in Greece and hence it seems that, following the tradition, in rural Greece the mother remains the core person in the family who chooses and prepares the food and thus has a major influence on adolescents' nutrition.

Eating out of home has also been identified as making an important contribution to adolescents' food choices. ${ }^{32}$ Our adolescents ate out of home either with family or with peers, with more healthful food being eaten with family and more 'junk type of food' with peers. It is possible that in this way, adolescents are able to achieve some kind of balance in their meals in terms of taste and healthfulness, but also some emotional balance by being accepted by their peers as well as being part of the family. Snacks purchased out of home generally have low nutrient value and are usually full of fat and sugar. ${ }^{33}$ This is evident in the findings of the current study, where foods such as burgers and fast-food type of food, sweets and soft drinks were consumed when adolescents ate a snack. 
About half of the adolescents purchased a snack daily from the school canteen and half of them had an afternoon snack out with peers: the latter foods are mostly available in school canteens and places where adolescents regularly meet. This dietary pattern may partly be the explanation for the low compliance with the Mediterranean diet as observed in the present and other studies from the area. Modification of foods at school canteens and in places where adolescents usually go can be instrumental in enhancing their nutritional quality.

Overall, in the present study girls were more likely than boys to make healthier food choices. In our study, girls consumed significantly more vegetables and fruits, took a home prepared snack to school more frequently, read significantly more details on food labels and had overall a better KIDMED score than boys did. These data indicate better nutrition knowledge in adolescent girls, a finding that has also been described elsewhere. ${ }^{34}$ In our study, for the observed gender differences other possible attributing factors might be the fact that girls skipped meals more frequently than boys and had more concerns about body weight and image, while boys by eating out with peers more frequently consumed more fastfood type of food. Boys are generally more active than girls, a factor that can also affect the amount of food consumed and the food choices. ${ }^{8}$ However, in our study we collected no data regarding the physical activity of adolescents.

It is noteworthy that a significant proportion of adolescents in our study consumed alcohol, although it is prohibited by law for people under the age of 18 . Previous researchers elsewhere ${ }^{35}$ have reached the same worrying conclusion, thus parents should be more careful and pay better attention to this point.

Some potential limitations in our study should be noted. First, no anthropometric measurements were obtained and thus it is not clear if adoption of weight concerns and dieting practices are based on actual weight or are part of the normal maturation process of adolescents. Second, the region studied is a mainly rural area and thus does not accurately represent the whole country, so that extrapolation of our data for all of Greece may not be appropriate. We believe, however, that the area shares the same sociodemographic and cultural characteristics as the majority of rural areas of mainland Greece and thus the results of this study may be considered as representative of rural Greece. Third, the food frequency questionnaires that have been used, although including foods more frequently consumed in Greece, should probably incorporate more detailed lists of foods that are more popular among adolescents. On the other hand, in adolescents, as well as in adults, there is no perfect method of assessing dietary intake. Finally, data on the physical activity of adolescents were not collected. These data might have been useful to explain further gender and age differences as well as certain eating behaviours.

\section{CONCLUSIONS}

Body weight concerns, dieting, food knowledge, parental control, maternal education, family meals, eating with peers, age and gender are factors that affect food choices among Greek adolescents. Despite the fact that certain traditions remain strong in rural Greece, globalization seems to be eroding them and to be leading to food modifications that are not compatible with the traditional Mediterranean diet and healthy eating. Understanding adolescents' perceptions towards food and nutrition along with factors that influence adolescent food behaviour might help to promote health among adolescents and improve quality of life.

None of the authors has any potential conflict of interest. No grants were received for this work.

\section{REFERENCES}

1. Roditis ML, Parlapani ES, Tzotzas T, Hassapidou M, Krassas GE, 2009 Epidemiology and predisposing factors of obesity in Greece: from the Second World War until today. J Pediatr Endocrinol Metab 22: 389-405.

2. Jackson-Leach R, Lobstein T, 2006 Estimated burden of paediatric obesity and co-morbidities in Europe. Part 1. The increase in the prevalence of child obesity in Europe is itself increasing. Int J Pediatr Ob 1: 26-32.

3. Baskin ML, Ard J, Franklin F, Allison DB, 2005 Prevalence of obesity in the United States. Obesity Reviews 6: 5-7.

4. Must A, Jacques PF, Dallal GE, et al, 1992 Longterm morbidity and mortality of overweight adolescents. N Engl J Med 327: 1350-1355.

5. Contento IR, Williams SS, Michela JL, Franklin AB, 2006 Understanding the food choice process of adolescents in the contex of family and friends. J Adolesc 
Health 38: 575-582.

6. Serra-Majem L, Ribas L, Ngo J, et al, 2004 Food, youth and the Mediterranean Diet in Spain. Development of KIDMED, Mediterranean Diet Quality Index in children and adolescents. Public Health Nutr 7: 931-935.

7. Greco L, Musmarra F, Franzese C, Auricchio S, 1998 Early childhood feeding practices in southern Italy: is the Mediterranean diet becoming obsolete? Study of 450 children aged 6-32 months in Campania, Italy. Cultural Paediatric Association. Acta Paediatr 87: 250-256.

8. Kontogianni MD, Vidra N, Farmaki AE, et al, 2008 Adherence Rates to the Mediterranean Diet Are Low in a Representative Sample of Greek Children and Adolescents. J Nutr 138: 1951-1956.

9. Kourbala G, Panagiotakos DB, Mihas K, et al, 2009 Dietary patterns in relation to socio-economics and lifestyle characteristic among Greek adolescents: a multivariate analysis. Public Health Nutr 12: 1366-1372.

10. Yannakoulia M, Karayannis D, Terzidou M, Kokkevi A, Sidossis LS, 2004 Nutrition-related habits of Greek adolescents. Eur J Clin Nutr 58: 580-586.

11. Gnardellis C, Trichopoulou A, Katsouyanni K, Polychronopoulos E, Rimm EB, Trichopoulos D, 1995 Reproducibility and validity of an extensive semiquantitative food frequency questionnaire among Greek school teachers. Epidemiology 6: 74-77.

12. Tsamita I, Kontogianni P, Karteroliotis K, 2007 Assessment of dietary habits in Greek adolescents. Inquiries in Sport \& Physical Education 5: 105-115.

13. Bilali A, Galanis P, Velonakis E, Katostaras T, 2010 Factors associated with abnormal eating attitudes among Greek adolescents. J Nutr Educ Behav 42: 292-298.

14. Van Strien T, van Niekerk R, Ouwens MA, 2009 Perceived parental food controlling practices are related to obesogenic or leptogenic child life style behaviours. Appetite 53: 151-154.

15. Anschutz DJ, Van Strien T, Engels RC, 2008 Exposure to slim images in mass media: television commercials as restriction in restrained eaters. Health Psychol 27: 401-408.

16. Derenne JL, Beresin EV, 2006 Body image, media and eating disorders. Acad Psychiatry 30: 257-261.

17. Ree M, Riediger N, Moghadasian MH, 2008 Factors affecting food selection in Canadian population. Eur $\mathrm{J}$ Clin Nutr 62: 1255-1262.

18. Striegel-Moore RH, Thompson D, Affenito SG et al, 2006 Correlates of beverage intake in adolescent girls: The national heart, lung, and blood institute growth and health study. J Pediatr 148: 183-187.

19. Papakonstantinou E, Hargrove JL, Huang CL, Crawley CC, Canolty NL, 2002 Assessment of perceptions of nutrition knowledge and disease using a group interactive system: the Perception Analyzer. J Am Diet Assoc 102: 1663-1668.

20. Lazarou C, Kalavana T, 2009 Urbanization influences dietary habits of Cypriot children:the CYKIDS study. Int J Public Health 54: 69-77.

21. Kafatos A, Diacatou A, Voukiklakis G, et al, 1997 Heart disease risk-factor status and dietary changes in the
Cretan population over the past 30 years. The seven countries study. Am J Clin Nutr 65: 1882-1886.

22. Huang TT, Kaur H, McCarter KS, Nazir N, Choi WS, Ahluwalia JS, 2004 Reading nutrition labels and fat consumption in adolescents. J Adolesc Health 35: 399401 .

23. McCullum C, Achterberg CL, 1997 Food shopping and label use behaviour among high school-aged adolescents. Adolescence 32: 181-197.

24. Feunekes GIJ, de Graff C, Meyboom S, van Staveren WA, 1998 Food choice and fat intake of adolescents and adults: associations of intakes within social networks. Prev Med 26: 645-656.

25. Neumark-Sztainer D, Story M, Ackard, et al, 2000 Family meals among adolescents: findings from pilot study. J Nutr Educ Behav 32: 335-340.

26. Neumark-Sztainer D, Larson NI, Fulkerson JA, Eisenberg ME, Story M, 2010 Family meals and adolescents: what have we learned from Project EAT (Eating Among Teens?). Public Health Nutr 13: 1113-1121.

27. Fulkerson JA, Kubic MY, Story M, Lytle L, Arcan C 2009 Are there nutrirional and other benefits associated with family meals among at risk youth? J Adolesc Health 45: 389-395.

28. Burgess-Champoux TL, Larson N, Neumark-Sztainer D, Hannan PJ, Story M, 2009 Are family meal patterns associated with overall diet quality during the transition from early to middle adolescence? J Nutr Educ Behav 41: 79-86.

29. Rogers I, Emmett P, ALSPAC Study Team, 2003 The effect of maternal smoking status, educational level and age on food and nutrient intakes in preschool children: results from the Avon Longitudinal Study of Parents and Children. Eur J Clin Nutr 57: 854-864.

30. Sausenthaler S, Kompauer I, Mielck A, et al, 2007 Impact of parental education and income inequality on children's food intake. Public Health Nutr 10: 24-33.

31. Riediger ND, Shooshtari S, Moghadasian MH, 2007 The influence of sociodemographic factors on patterns of fruit and vegetable consumption in Canadian adolescents. J Am Diet Assoc 107: 1511-1518.

32. Fitzgerald A, Heary C, Kelly C, Nixon E, Shevlin M, 2012 Self-efficacy for healthy eating and peer support for unhealthy eating are associated with adolescents'food intake patterns. Appetite 63: 48-45.

33. Kerr MA, Rennie KL, McCaffrey TA, Wallace JM, Hannon-Fletcher MP, Livingstone MB, 2009 Snacking patterns among adolescents: a comparison of type, frequency and portion size between Britain in 1997 and Northern Ireland in 2005. Br J Nutr 101: 122-131.

34. Pirouznia M, 2001 The association between nutrition knowledge and eating behavior in male and female adolescents in the US. Int J Food Sci Nutr 52: 127-132.

35. Lew K, Barlow PJ, 2005 Dietary practices of adolescents in Singapore and Malaysia Singapore Med J 46: 282-288. 